\title{
The Luminous Type Ibc Supernova 2010as
}

\section{Gastón Folatelli}

Kavli Institute for the Physics and Mathematics of the Universe, Todai Institutes for Advanced Study, the University of Tokyo, Kashiwa, Japan 277-8583 email: gaston.folatelli@ipmu.jp

On behalf of the MCSS, the CSP, and collaborators

\begin{abstract}
We present photometric and spectroscopic follow-up observations of SN 2010as carried out by the MCSS and CSP. The SN appears to be of the transitional type Ibc (SN Ibc) and is spectroscopically similar to the peculiar SN 2005bf. Based on distance and extinction estimates, a bolometric luminosity light curve is constructed showing that this was a relatively luminous SN Ibc. He I line expansion velocities are remarkably low and remain nearly constant with time, similarly to SN 2005bf. A preliminary model is presented with a progenitor ZAMS mass of $15 M_{\odot}$ and a large yield of $0.35 M_{\odot}$ of ${ }^{56} \mathrm{Ni}$.
\end{abstract}

Keywords. supernovae: general, supernovae: individual (SN 2010as)

\section{Photometry}

SN 2010as was discovered in NGC 6000 on March 19.2 UT by the CHilean Automatic Supernova sEarch (CHASE; Maza et al. 2010). Follow-up in $B V R I g^{\prime} r^{\prime} i^{\prime} z^{\prime}$ bands was obtained with the 40-cm PROMPT 1, 3 and 4 telescopes at CTIO (Figure 1, left panel). The SN was caught 10 days before $B$-band maximum light and followed until $\approx 100$ days after.

Observed colors corrected for Galactic reddening of $E(B-V)_{\mathrm{MW}}=0.17 \mathrm{mag}$ (from $\mathrm{NED} \dagger$ ) were compared with those of a sample of SNe Ib and Ic from the CSP (Stritzinger et al. , in prep.) to derive a host-galaxy reddening of $E(B-V)_{\text {host }}=0.35$ mag $\ddagger$. Adopting a distance of $32.7 \pm 3.6 \mathrm{Mpc}$ to the host (NED), reddening-free absolute peak magnitudes of $M_{B}=-18.1 \pm 0.5 \mathrm{mag}$ and $M_{V}=-18.5 \pm 0.4 \mathrm{mag}$ are obtained, indicating that SN 2010as was a relatively luminous SN Ibc.

\section{Spectroscopy}

A spectral time series between -10 and +309 days relative to $B$-band maximum was obtained by the MCSS and CSP with instruments from Las Campanas, ESO Paranal, SOAR and Gemini Observatories (see left panel of Figure 2). The pre-maximum spectra are of type Ic, dominated by Ca II and Fe II lines, with possible $\mathrm{H} \alpha$ or Si II at $\approx 6200$ $\AA$. Then He I lines develop marking the transition to a SN Ib. The right panel of Figure 2 compares the spectra with those of SNe 2005bf (Folatelli et al. 2006) and 2007Y (Stritzinger et al. 2009) at about one week before maximum light, around the time of maximum, and three weeks after. The three SNe are very similar before maximum, but then SN 2007Y develops strong He I lines. The other two SNe remain similar, with SN 2010as showing stronger Ca II features. An additional similarity with SN 2005bf is the peculiarly low He I line velocities observed at all times.

$\dagger$ NASA/IPAC Extragalactic Database

$\ddagger$ This agrees with the value derived from a Na I D equivalent width of $2.1 \AA$ measured on X-Shooter spectra. 

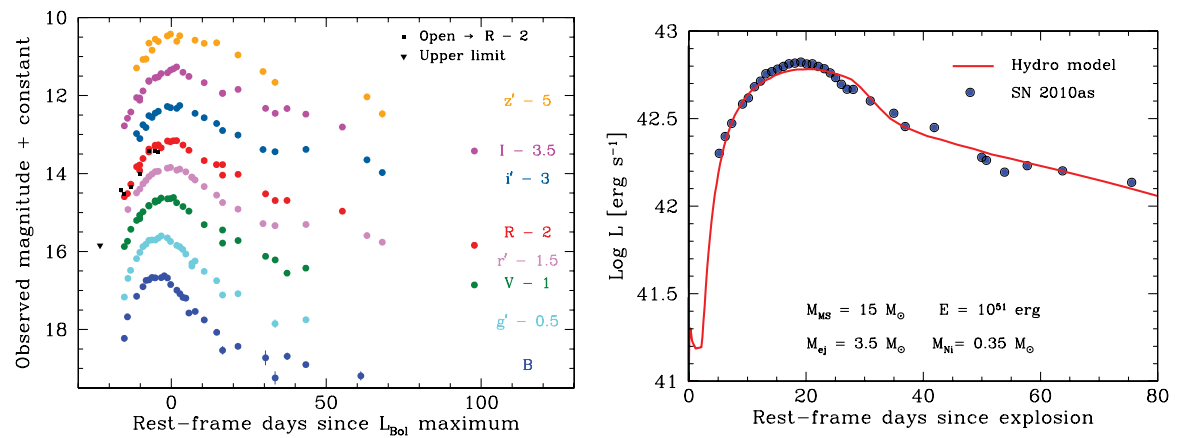

Figure 1. (Left) $B V R I g^{\prime} r^{\prime} i^{\prime} z^{\prime}$ light curves. (Right) Bolometric luminosity for a model (solid line) with the indicated physical parameters, compared with the data (dots).
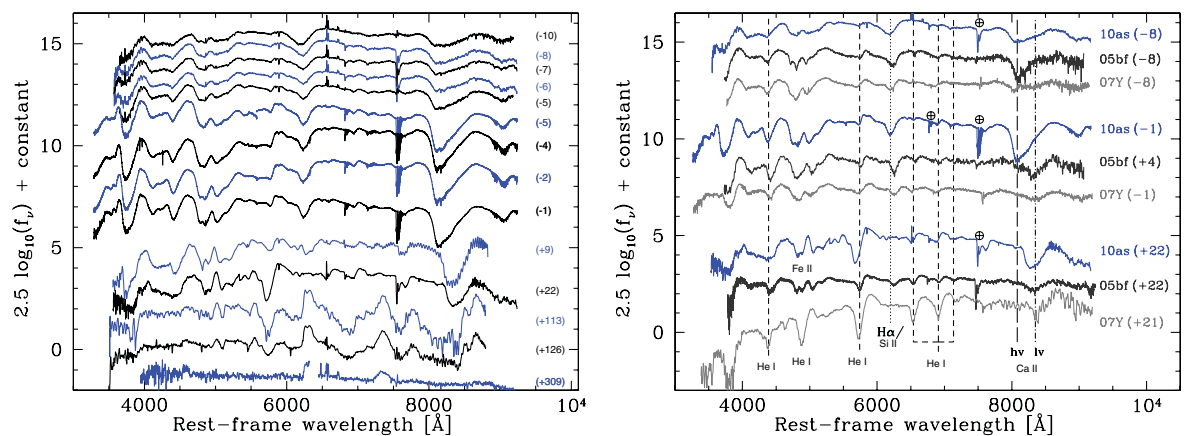

Figure 2. (Left) Spectroscopic time series. (Right) Spectral comparison with SNe Ibc 2005bf and $2007 \mathrm{Y}$ at three different epochs.

\section{Physical parameters}

A bolometric light curve was obtained by integrating the extinction-corrected optical flux and adding the extrapolation of a black-body fit toward the IR, and a straight line in the UV from the $B$-band point to zero flux at $2000 \AA$. The extrapolated UV+IR flux accounts for up to $50 \%$ of the total estimated flux. Assuming the extinction derived in Section 1, the resulting peak luminosity is $L_{\mathrm{Bol}}=6.3 \times 10^{42} \mathrm{erg} \mathrm{s}^{-1}$. This is comparable with the cases of the peculiar SNe 2005bf and 2009bb, which makes SN 2010as a relatively luminous Ibc event.

A preliminary hydrodynamical model (Bersten et al. 2011) was computed to reproduce the bolometric luminosity (see assumed physical parameters in the right panel of Figure 1). A relatively large amount of ${ }^{56} \mathrm{Ni}, M_{\mathrm{Ni}}=0.35 M_{\odot}$, is required to explain the luminous peak. The explosion energy of $E=10^{51} \mathrm{erg}$, however, overestimates the measured expansion velocities. This may be solved by assuming larger progenitor and ejected masses.

\section{References}

Bersten, M. C., et al. 2011, ApJ, 729, 61

Folatelli, G., et al. 2006, ApJ, 641, 1039

Maza, J., et al. 2010, CBET, 2215, 1

Stritzinger, M., et al. 2009, ApJ, 696, 713

Stritzinger, M., et al. 2012 in prep. 